\title{
CHARACTERIZATION OF SMALL SUPERNUMERARY MARKER CHROMOSOMES BY A SIMPLE MOLECULAR AND MOLECULAR CYTOGENETICS APPROACH
}

\author{
Liehr $\mathrm{T}^{1, *}$, Trifonov $\mathrm{V}^{1,2}$, Polityko $\mathrm{A}^{1,3}$, Brecevic $\mathrm{L}^{1,4}$, Mrasek $\mathrm{K}^{1}$, Weise $\mathrm{A}^{1}$, \\ Ewers $E^{1}$, Reich $D^{1}$, Iourov ${ }^{1,5}$, Mkrtchyan $\mathrm{H}^{1,6}$, Manvelyan $\mathrm{M}^{1,6}$, Kosyakova $\mathrm{N}^{1,7}$
}

*Corresponding Author: Dr. Thomas Liehr, Institut für Humangenetik und Anthropologie, Kollegiengasse 10, D-07743 Jena, Germany; Tel.: +49-3641-935533; Fax: +49-3641-935582; E-mail: i8lith@mti.uni-jena.de

\section{ABSTRACT}

Small supernumerary marker chromosomes (sSMC) are still a major problem especially in prenatal cytogenetic diagnostics and counseling. These structurally abnormal chromosomes cannot be identified or characterized unambiguously by conventional banding cytogenetics alone, and are generally about the size of or smaller than a chromosome 20 in the same metaphase spread. We describe a straightforward algorithm, based on data from 2,211 reported cases (http://www. markerchromosomes.ag.vu) to quickly characterize the sSMC's chromosomal origin.

Key words: Small supernumerary marker chromosomes (sSMC); Cytogenetic(s); Fluorescence in situ hybridization (FISH); Metaphase FISH, Molecular cytogenetics; Prenatal diagnostics.

\footnotetext{
1 Institute of Human Genetics and Anthropology, Friedrich-Schiller-University Jena, Germany

${ }^{2}$ Institute of Cytology and Genetics, Novosibirsk, Russia

${ }^{3}$ Institute of Hereditary Diseases, Minsk, Belarus

${ }^{4}$ School of Medicine, Croatian Institute for Brain Research, Zagreb, Croatia

${ }^{5}$ National Research Center of Mental Health, Russian Academy of Medical Sciences, Moscow, Russia

${ }^{6}$ Department of Genetic and Laboratory of Cytogenetics, State University, Jerewan, Armenia

${ }^{7}$ Research Centre for Medical Genetics, Russian Academy of Medical Sciences, Moscow, Russian Federation
}

\section{INTRODUCTION}

Small supernumerary marker chromosomes (sSMC) are present in $\sim 2.7$ million people worldwide [1]. They are defined as structurally abnormal chromosomes that cannot be identified or characterized unambiguously by conventional banding cytogenetics alone; they are generally equal in size or smaller than a chromosome 20 in the same metaphase spread. Additionally, sSMC can be present in an otherwise normal karyotype, in a numerically abnormal karyotype (e.g., Turner's or Down's syndromes) or in a structurally abnormal but balanced karyotype with or without ring chromosome formation. If detected in banding cytogenetics, they are a major problem as they are too small to be characterized for their chromosomal origin or content by traditional banding techniques. Molecular cytogenetic techniques are necessary for their characterization [2]. Cases with a de novo SSMC, particularly those that are prenatally ascertained, are not easy to correlate with a clinical outcome [3]. It has been established that substantial parts of sSMC lead to four specific syndromes, i.e., Pallister-Killian $[=\mathrm{i}(12 \mathrm{p})]$, isochromosome $18 \mathrm{p}[\mathrm{i}(18 \mathrm{p})]$, cat-eye $[\mathrm{i}(22 \mathrm{p} \sim \mathrm{q})]$ and derivative chromosome 22 [der(22) $\mathrm{t}(11 ; 22)]$ syndromes [2]. In general, the risk for an abnormal phenotype in prenatally ascertained de novo cases with $\mathrm{sSMC}$ is considered to be $\sim 13 \%$ [4]. This has been refined to $7 \%$ (for sSMC from chromosomes 13, 14, 21 or 22 ) and $28 \%$ (for all non-acrocentric autosomes) [5] and has recently been suggested to be $26-30 \%[1,6]$. Also, 
generally speaking, sSMC transmitted by normal sSMC carriers to their progeny are not correlated with clinical problems [7], although exceptions have been described [8].

\section{MATERIALS AND METHODS}

By reviewing the literature, all available reported sSMC cases (total 2,211) were collected on a regularly updated sSMC homepage [9]. All cases were included when at least the chromosomal origin of an sSMC was reported in an article listed at the National Center for Biotechnology Information webpage (or If available, gender of the carrier, age at diagnosis, the cytogenetically studied material (blood, amniocytes, fibroblasts), parental origin of SSMC, the GTG-banding result including mosaicism of sSMC, final fluorescence in situ hybridization (FISH) results of sSMC, applied FISH methods, exclusion of uniparental disomy (UPD) for the sSMC's sister-chromosomes and the clinical symptoms are also listed. Thus, according to this data [9], the distribution of the chromosomal origin of sSMC is summarized as available from the literature (Fig. 1). Even though this data is biased by the fact that not all but mainly 'the interesting SSMC cases' are published, it provides the only available insight on the chromosomes most frequently involved in SSMC-formation.
According to that data, an algorithm for a straightforward characterization of SSMC origins was worked out (as presented in Results). According to the chromosome-specific frequency of sSMC, the application of commercially available centromere specific probes is suggested to quickly achieve information on the chromosomal origin of a centric sSMC [2]. Commercially available whole chromosome painting probes can be used for the characterization of neocentric sSMC. Neocentric, also called analphoid SSMC, "carry newly derived centromeres (or "neocentromeres") that are apparently formed within interstitial chromosomal sites that have not previously been known to express centromere function" [10].

\section{RESULTS}

The chromosomal distribution of sSMC from 2,211 reported cases is shown in Fig. 1. The four syndromes Pallister-Killian, isochromosome 18p, cat-eye and derivative chromosome 22 syndromes, accounted for $31 \%$ of cases. Overall, chromosome \#15 was the most frequent participant $(30 \%)$ and was followed by chromosomes \#14/\#22 (26\%), \#12 (9\%), \#18 (7\%), \#13/\#21 (5\%), \#8 (4\%), \#1 ( 2\%), \#16 ( 2\%), \#9 ( 1\%), \#3 ( 1\%), \#20 ( 1\%), X $(\sim 1 \%)$, and the remainder $(\sim 11 \%)$. Interestingly, a very similar distribution is observed in neocentric

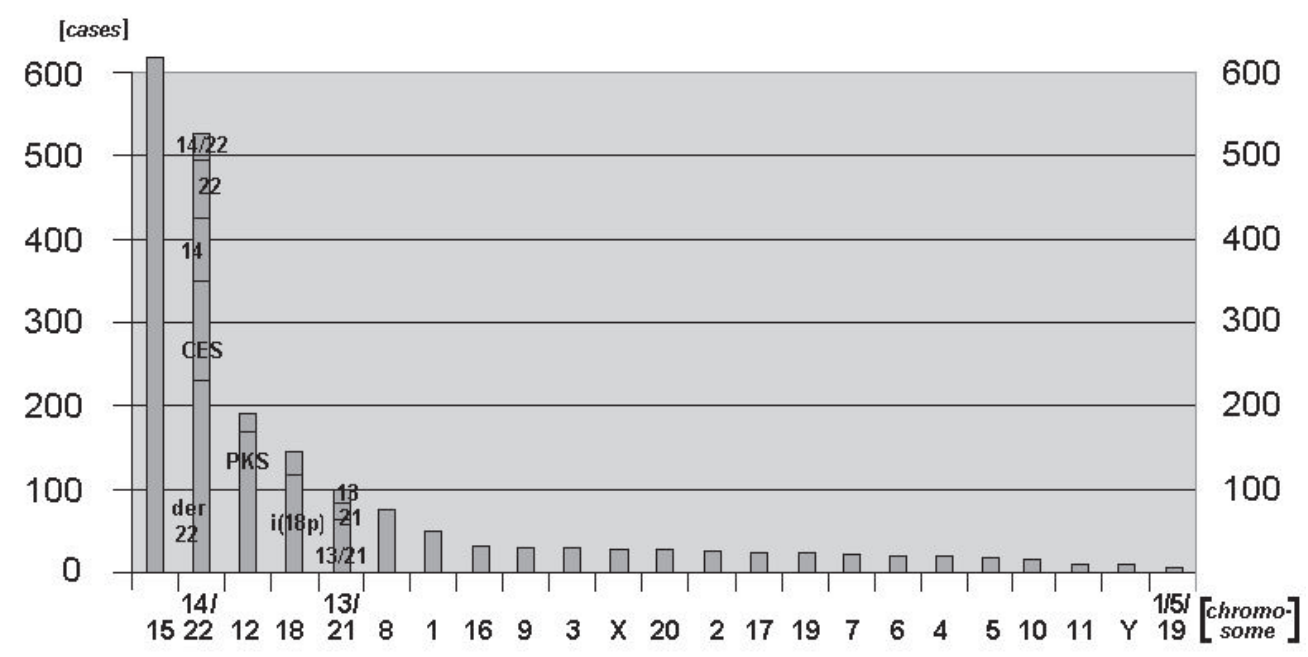

Figure 1. Frequency of sSMC according to their chromosomal origin: Chromosomal origin of 2,211 sSMC cases collected from the literature [9]. From left to right the most frequent to the most rare. Abbreviations: CES: cat-eyesyndrome; der 22: derivative chromosome 22 syndrome; i(18p): isochromosome 18p syndrome; PKS: Pallister Killian syndrome. 
sSMC: chromosome \#15 (24\%), chromosomes \#8 (15\%), \#13 (15\%), \#8 (15\%), \#3 (9\%),\#1 (7\%), \#12 (4\%), which could be in connection with the mechanisms of sSMC-formation (U-type exchange, summarized in [2]).

Comprehensive characterization of marker chromosomes is often hampered by the lack of available multicolor-FISH (M-FISH) approaches (such as micro dissection and reverse FISH) [11], M-FISH applying whole chromosome painting probes (i.e., M-FISH [12], spectral karyotyping (SKY) [13], or (sub)centromere-specific probes (cenM-FISH) [14] and subcenM-FISH) [15]). Thus, an algorithm was developed that allows determination of the chromosomal origin of an sSMC in a straightforward and effective manner. The chromosomal origin of an $\mathrm{SSMC}$ provides better risk assessment of the clinical outcome based on similar cases summarized on the sSMC homepage [9]. A first attempt at genotype-phenotype correlation for sSMC has been reported [16].

The following algorithm provides a practical method for determining the chromosomal origin of an sSMC for diagnostic laboratories which do not have sophisticated molecular cytogenetic possibilities:

1) Clarify by conventional chromosome-banding analysis of parental peripheral blood if sSMC is de novo. If the sSMC is inherited go to 2); if it is de novo or its parental origin cannot be determined go to 3$)$.

2) If the sSMC is inherited from one clinically normal parent, the identification of the sSMC origin may be replaced by genetic counseling with close monitoring of the pregnancy by high resolution ultrasound examinations. However, an inherited sSMC may be connected with clinical abnormalities in exceptional cases ([8] case I). If origin of the sSMC is to be clarified, go to 4).

$3)$ If the de novo sSMC is almost the size of chromosome 20 in the same metaphase spread, the presence of a large inverted duplication chromosome 15 (inv dup15), an isochromosome 18 [i(18p)] or an $[i(12 p)]$ should be excluded by the appropriate centromeric and/or whole chromosome painting probes. If, as happens in about one-third of cases, the origin of the sSMC is clarified in that way, go to 9). If the origin of the $\mathrm{SSMC}$ was not determined in this way, go to 4).
4) If a clear and positive nucleolus organization region (NOR) silver staining result is obtained [17] for the SSMC, its origin can be determined by hybridizing commercially available centromeric probes for all acrocentric chromosomes, i.e., $\# 13 / \# 21, \# 14 / \# 22$, \#15. If, as happens in $~ 75 \%$ of cases, the origin of the sSMC is clarified in this way, go to 9). If the origin of the sSMC was not determined in this way, go to $\mathbf{5}$ ).

5) To determine the origin of the sSMC use commercially available centromeric probes, testing sequentially for $\# 8, \# 1, \# 20, \mathrm{X}, \# 18, \# 3$ and $\# 12$. Even if sSMC was NOR-negative, test for \#14/\#22, $\# 15$ and \#13/\#21, as cases have been reported with sSMC derived from acrocentric chromosomes, but without NOR [9]. If, as happens in $\sim 90 \%$ of cases, the origin of the $\mathrm{SSMC}$ is clarified in this way, go to 9). If the origin of the $\mathrm{SSMC}$ was not determined in this way, go to 6 ).

6) To determine if the case is a rare one with a neocentric sSMC, a commercially available pan centromeric probe should be used. This test is important since neocentric sSMC nearly always have a clinically adverse prognosis $[2,9,16]$. In $\sim 4 \%$ of the cases no $\alpha$-satellite DNA is present on the sSMC. If the sSMC has $\alpha$-satellite DNA, go to 7); if the sSMC has no $\alpha$-satellite DNA, go to 8).

7) An sSMC with $\alpha$-satellite DNA can still arise from 12 different human chromosomes. If there is enough material to continue the analysis, proceed in the following sequence (applying centromeric probes if nothing else is mentioned): \#19 (whole chromosome painting probe), \#9, \#16, \#17, \#7, \#6, $\# 2$, \#4, \#5 (whole chromosome painting probe), \#11 and Y. If, as happens in $\sim 100 \%$ of cases, the origin of a centric sSMC is clarified, go to 9).

8) To characterize the origin of a neocentric sSMC use FISH, applying the following sequence of whole chromosome painting probes: $\# 15, \# 8, \# 13$, $\# 3$, \#1 and \#12. If, as happens in $~ 75 \%$ of cases, the origin of the neocentric SSMC is clarified, go to 9).

9) In $\sim 10 \%$ of $\mathrm{sSMC}$ cases, UPD of the cytogenetically normal sSMC's sister chromosome has been reported [2]. Because of this, it has been recommended [2,18-19] that, after identification of the origin of the SSMC, the normal sister chromosomes should be tested for their parental origin to exclude possible UPD. This can be tested by molecular genetic approaches, such as 
microsatellite analysis [19] or methylation-specific polymerase chain reaction (PCR) [20] and should be done for every sSMC case in which parental cell material is available. Apart from the chromosomes known to be connected with imprinting (\#6, \#7, \#14, $\# 15$, \#20), other chromosomes should be tested as uniparental isodisomy can lead to homozygotization of an otherwise recessive, disease-causing gene (e.g., see [21]).

\section{DISCUSSION}

Our straightforward algorithms for characterization of the origin of an SSMC has the advantages that it can be performed without the use of sophisticated or highly specialized equipment, except for one- or two-color-FISH and that it provides the chromosomal origin of a sSMC. Moreover, if molecular genetic approaches such as micro satellite analysis are available, a possible disease-causing UPD can be excluded. However, only subcenMFISH [15] or array-CGH (comparative genomic hybridization) [22] can exclude or detect a small partial tri- or polysomy of the centromere-near region of the sSMC. In most cases, after exclusion of an UPD of an SSMC's sister chromosome, such an imbalance determines the clinical effect of the marker chromosome [16]. When possibilities for determination of the euchromatic contents of an sSMC are not available, all reported sSMC cases (sorted by chromosomal origin) can be found on the sSMC homepage [9]. The authors welcome the opportunity to characterize sSMC cases by subcenM-FISH or other approaches.

\section{ACKNOWLEDGMENTS}

This study was supported in part by funds from the DFG (436 RUS 17/135/03; 436 RUS 17/109/04, 436 RUS 17/22/06, 436 WER 17/1/04, 436 WER $17 / 5 / 05$, WE 3617/2-1), the Schering Foundation, the Boehringer Ingelheim Fonds and the Evangelische Studienwerk e.V. Villigst.

\section{REFERENCES}

1. Liehr T, Weise A. Frequency of small supernumerary marker chromosomes in prenatal, newborn, developmentally retarded and infertility diagnostics. Int J Mol Med 2007; 19(5): 719-731.
2. Liehr T, Claussen U, Starke H. Small supernumerary marker chromosomes (sSMC) in humans. Cytogenet Genome Res 2004; 107(1-2): 55-67.

3. Paoloni-Giacobino A, Morris MA, Dahoun SP. Prenatal supernumerary $\mathrm{r}(16)$ chromosome characterized by multiprobe FISH with normal pregnancy outcome. Prenat Diagn 1998; 18(7): 751-752.

4. Warburton D. De novo balanced chromosome rearrangements and extra marker chromosomes identified at prenatal diagnosis: clinical significance and distribution of breakpoints. Am J Hum Genet 1991; 49(5): 995-1013.

5. Crolla JA. FISH and molecular studies of autosomal supernumerary marker chromosomes excluding those derived from chromosome 15: II. Review of the literature. Am J Med Genet 1998; 75(4): 367-381.

6. Graf MD, Christ L, Mascarello JT, Mowrey P, Pettenati M, Stetten G, Storto P, Surti U, Van Dyke DL, Vance GH, Wolff D, Schwartz S. Redefining the risks of prenatally ascertained supernumerary marker chromosomes: a collaborative study. J Med Genet 2006; 43(8): 660-664.

7. Bröndum-Nielsen K, Mikkelsen M.; A 10-year survey, 1980-1990, of prenatally diagnosed small supernumerary marker chromosomes, identified by FISH analysis. Outcome and follow-up of 14 cases diagnosed in a series of 12,699 prenatal samples. Prenat Diagn 1995; 15(7): 615-619.

8. Anderlid BM, Sahlen S, Schoumans J, Holmberg E, Ahsgren I, Mortier G, Speleman F, Blennow E. Detailed characterization of 12 supernumerary ring chromosomes using microFISH and search for uniparental disomy. Am J Med Genet 2001; 99(3): 223-233.

9. Liehr, T. sSMC homepage (http://www. markerchromosomes.ag.vu).

10. Choo KH. Centromere DNA dynamics: latent centromeres and neocentromere formation. Am J Hum Genet 1997; 61(6): 1225-1233.

11. Starke H, Raida M, Trifonov V, Clement JH, Loncarevic IF, Heller A, Bleck C, Nietzel A, Rubtsov N, Claussen U, Liehr T. Molecular cytogenetic characterisation of an acquired minute supernumerary marker chromosome as the sole abnormality in a case clinically diagnosed as atypical Philadelphia-negative chronic myelogenous leukaemia. Br J Haematol 2001; 113(2): 435-438.

12. Speicher MR, Gwyn Ballard S, Ward DC. Karyotyping human chromosomes by combinatorial 

Reich $\mathrm{D}^{1}$, Iourov $\mathrm{I}^{1,5}$, Mkrtchyan $\mathrm{H}^{1,6}$, Manvelyan $\mathrm{M}^{1,6}$, Kosyakova $\mathrm{N}^{1,7}$

multi-fluor FISH. Nat Genet 1996; 12(4): 368-375.

13. Schröck E, du Manoir S, Veldman T, Schoell B, Wienberg J, Ferguson-Smith MA, Ning Y, Ledbetter DH, Bar-Am I, Soenksen D, Garini Y, Ried T. Multicolor spectral karyotyping of human chromosomes. Science 1996; 273(5274): 494-497.

14. Nietzel A, Rocchi M, Starke H, Heller A, Fiedler W, Wlodarska I, Loncarevic IF, Beensen V, Claussen U, Liehr T. A new multicolor-FISH approach for the characterization of marker chromosomes: centromere-specific multicolor-FISH (cenM-FISH). Hum Genet 2001; 108(3): 199-204.

15. Starke H, Nietzel A, Weise A, Heller A, Mrasek K, Belitz B, Kelbova C, Volleth M, Albrecht B, Mitulla B, Trappe R, Bartels I, Adolph S, Dufke A, Singer S, Stumm M, Wegner RD, Seidel J, Schmidt A, Kuechler A, Schreyer I, Claussen U, von Eggeling F, Liehr T. Small supernumerary marker chromosomes (SMCs): genotype-phenotype correlation and classification. Hum Genet 2003; 114(1): 51-67.

16. Liehr T, Mrasek K, Weise A, Dufke A, Rodriguez L, Martinez Guardia N, Sanchis A, Vermeesch JR, Ramel C, Polityko A, Haas OA, Anderson J, Claussen U, von Eggeling F, Starke H. Small supernumerary marker chromosomes-progress towards a genotype-phenotype correlation. Cytogenet Genome Res 2006; 112(1-2): 23-34.
17. Bloom SE, Goodpasture C. An improved technique for selective silver staining of nucleolar organizer regions in human chromosomes. Hum Genet 1975; 34(2): 199-206.

18. Kotzot D. Advanced parental age in maternal uniparental disomy (UPD): implications for the mechanism of formation. Eur J Hum Genet 2004; 12(5): 343-346.

19. Salafsky IS, MacGregor SN, Claussen U, von Eggeling F. Maternal UPD 20 in an infant from a pregnancy with mosaic trisomy 20. Prenat Diagn 2001; 21(10): 860-863.

20. Nietzel A, Albrecht B, Starke H, Heller A, Gillessen-Kaesbach G, Claussen U, Liehr T. Partial hexasomy 15 pter $\rightarrow 15 \mathrm{q} 13$ including SNRPN and D15S10: first molecular cytogenetically proven case report. J Med Genet 2003; 40(3): e28.

21. Thompson DA, McHenry CL, Li Y, Richards JE, Othman MI, Schwinger E, Vollrath D, Jacobson SG, Gal A. Retinal dystrophy due to paternal isodisomy for chromosome 1 or chromosome 2, with homoallelism for mutations in RPE65 or MERTK, respectively. Am J Hum Genet 2002; 70(1): 224-229.

22. Liehr T, Starke H, Senger G, Melotte C, Weise A, Vermeesch JR. Overrepresentation of small supernumerary marker chromosomes (sSMC) from chromosome 6 origin in cases with multiple sSMC. Am J Med Genet A 2006; 140(1): 46-51. 